NOTA CORTA
Rev Colombiana Cienc Anim 2016; 8(1):88-93.
EFECTOS DE LA COMPACTACION DE SUELOS POR EL PISOTEO
DE ANIMALES, EN LA PRODUCTIVIDAD DE LOS SUELOS.
REMEDIACIONES

\title{
EFFECTS OF SOIL COMPACTION BY TRAMPLING OF ANIMALS IN SOIL PRODUCTIVITY. REMEDIATIONS
}

\author{
MEDINA M. CARLOS,${ }^{1}$ M.Sc.
}

${ }^{1}$ Servicio Nacional de Aprendizaje (SENA). Instructor asesor. Geotecnia en Ingeniería Civil. Bogotá, Colombia

\section{Key words:}

\section{Soils,} grazing, trampling, compaction, soil structure, degradation, subsoilers

\begin{abstract}
Among the problems of physical degradation, compaction and densification are the main ones, and they cause strong decreases in subsequent crop yields thereof. The degradation of soil structure is a worldwide problem that can occur in all soils and livestock production levels. Given the above, this paper aims to analyze the main changes in the internal structure of the soil as a result of compaction by trampling of animals. Soil compaction due to the collapse or decrease in pore spaces, is the most common cause of physical restraint to growth and root development. As an aggravating factor, degradation of soil structure is regarded as the type of land degradation more difficult to locate and retrain; the reason is that this degradation is a subsurface phenomenon. Finally it is to establish some possible solutions to this problem, including the main method of restoring the porosity of the boundary layers of root growth is to apply biological methods as used, for example, the roots of natural vegetation or crops coverage, planted to act as biological subsoilers that penetrate dense horizons.
\end{abstract}

\section{Palabras Clave:}

\section{Suelos,} pastoreo, pisoteo, estructura del suelo, degradación, subsolador.

\section{Resumen}

Entre los problemas de la degradación física de los suelos, la compactación y el adensamiento son los principales, y estos causan fuertes decrementos en los rendimientos posteriores de los cultivos de los mismos. La degradación de la estructura del suelo es un problema mundial que puede ocurir en todos los suelos y niveles de producción pecuaria. Teniendo en cuenta lo anterior, este documento pretende analizar los principales cambios producidos en la estructura intema de los suelos como resultado de su compactación por el pisoteo de animales. La compactación del suelo, debido al colapso o disminución de los espacios de poros, es la causa más común de restricción física para el crecimiento y desarrollo de las raíces. Como agravante, la degradación de la estructura del suelo es comúnmente considerada como el tipo de degradación de tierras más dificil de localizar y reconvertir, la razón es que ésta degradación es un fenómeno subsuperficial. Finalmente se trata de establecer algunas posibles soluciones a dicha problemática, entre las cuales el método principal de restauración de la porosidad de las capas limitantes del crecimiento de las raíces es aplicar métodos biológicos como utilizar por ejemplo, las raíces de la vegetación natural o de cultivos de cobertura, plantados para que actúen como subsoladores biológicos que penetren en los horizontes densos. 


\section{Introducción}

El suelo es una de las partes fundamentales de las haciendas y terrenos productivos de la región, por lo que éste se convierte en elemento principal en el desarrollo de sus sistemas agroecológicos, agrícolas y pecuarios. El estudio de la compresión del suelo debido al pisoteo, por parte de los animales, durante el pastoreo es un tema de gran importancia en lo referente a la productividad actual y futura de los mismos.

Según BRADFORD y GUPTA (1986) la compresión del suelo consiste en la disminución de su volumen por la aplicación de una alta carga. Cuando este proceso ocurre sobre suelos saturados (todos los espacios vacíos del suelo llenos de agua) se denomina consolidación, y compactación cuando esta ocurre sobre suelos parcialmente saturados. En el primero de los casos la reducción de vacíos se da por la salida de agua de los espacios vacíos y en el segundo casos por la salida del aire.

RUSSELL (1977) considera que la compactación de los suelos reduce significativamente el volumen de los vacíos de un suelo, causando con ello alteraciones en su humedad y el posible intercambio de gases entre el suelo y la atmósfera, así como el libre desarrollo de las raíces.

DOUGLAS (1999) establece que la compactación del suelo se puede desarrollar no sólo en las grandes fincas mecanizadas, sino también por actividades de la labranza manual, y por las acciones del pastoreo y pisoteo animal. La compactación a menudo reduce las dimensiones de los poros lo suficiente como para inhibir la penetración de las raíces pero no suficientemente como para afectar el drenaje del agua a través del suelo; sin embargo, esa reducción puede reducir de manera significativa la velocidad o tasa de infiltración de la lluvia. Si un poro se reduce diez veces de tamaño, la cantidad de agua que puede fluir a través del mismo en un tiempo dado será diez mil veces menor en comparación con el diámetro inicial del poro. Esto constituye un efecto ambiental perjudicial de la compactación sobre la tasa de percolación y recarga de los acuíferos, eventualmente subyacentes, y sobre la sostenibilidad de los cultivos y de la producción agropecuaria en general. A la vez, es un efecto que redundará en fuertes velocidades superficiales que puede conllevar a procesos erosivos y de instabilidad de los suelos en el ecosistema en explotación.

El pisoteo de los animales durante el proceso de pastoreo, favorece la compactación de los suelos ubicados en el horizonte superior del suelo, afectando el hábitat de los microorganismos del suelo y la productividad de la pastura. Un ejemplo, es la destrucción del hábitat de las plantas leguminosas (Fabaceae spp.) que incluye a las bacterias fijadoras del nitrógeno atmosférico N2 (Rhizobium spp.) en las actividades vitales del ciclo biogeoquímico en la generación de nutrientes para las comunidades productoras primarias.

El problema de la degradación de tierras agrícolas a nivel mundial, clama por el desarrollo de nuevas tecnologías y sistemas de cultivo que conduzcan a una agricultura sostenible asentada en suelos sostenibles (AMÉZQUITA, 1998b).

Estructura del suelo: Es la arquitectura del suelo, o sea, la forma en que están ordenados las partículas sólidas y los espacios. La descripción de la estructura del suelo se refiere al tamaño y forma de las unidades de suelo o agregados y los espacios de aire o poros dentro y entre ellos. Los agregados están constituidos por partículas de suelo individuales ligados con materia orgánica, arcilla y hierro. Diferentes suelos tienen diferentes tipos de estructuras formadas en el transcurso de los años como una parte natural del proceso de la formación del suelo.

Los espacios de aire entre y dentro de las unidades son los más importantes. No solo distribuyen el aire, el agua y los nutrientes a través del suelo, sino que además son usados por las raíces de las plantas para anclar y sostener un cultivo sano. Los buenos suelos tienen una mezcla de microporos y macroporos: los macroporos para la entrada de agua y el drenaje, los microporos para el almacenamiento del agua.

Cambios en el suelo: Los efectos causados por el tránsito y el pisoteo de animales afectan primordialmente a la estructura del suelo pastoreado; o sea lo que se entiende por el ordenamiento espacial de los sólidos y poros del suelo (Fig.1).

Presiones de compactación ejercidas por la hacienda: El efecto del tránsito de hacienda está relacionado con las presiones ejercidas sobre el suelo, lo cual es función de la masa del animal, el tamaño de su pezuña y la energía cinética. Los datos de la Tabla 1 indican que las presiones ejercidas por ovinos, cuando están parados, promedian los $66 \mathrm{kPa}$ y los vacunos 138 $\mathrm{kPa}$. Estas presiones son equiparables a las ejercidas por rodillos de tractores no cargados (74 - $81 \mathrm{kPa}$ ), y las presiones de tracción ( $58 \mathrm{kPa}$ ) de vehículos arrastrados (Tabla 1). 


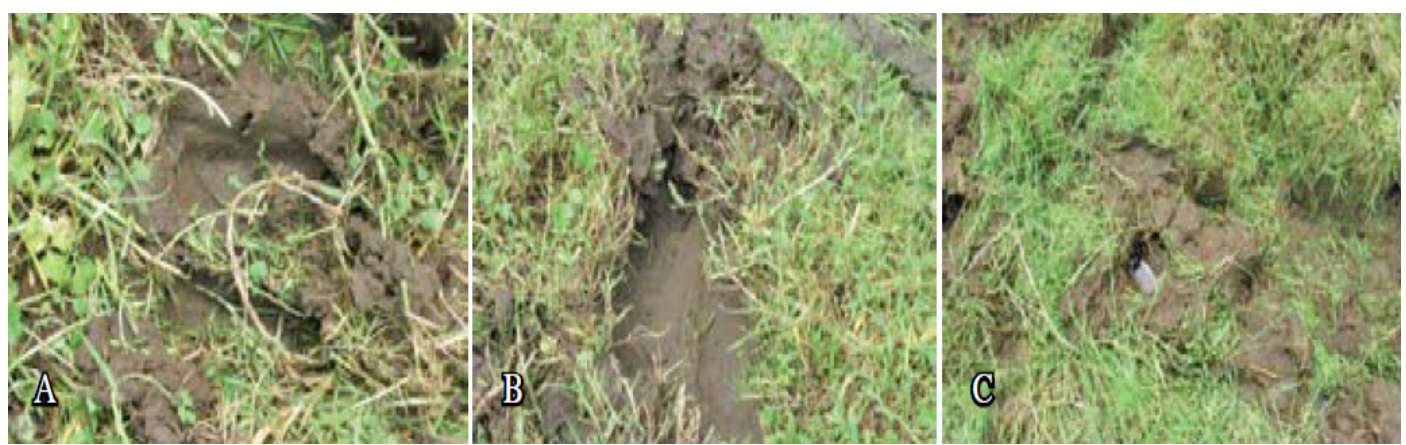

Figura 1. Efecto inmediato del pisoteo del ganado en la superficie del suelo con cobertura permanente de pasto kikuyo (Pennisetum clandestinum) (A. Huella de la pisada, B. Deslizamiento de la pisada y C. Acumulación de agua en área de pisoteo). Coronado, San José, Costa Rica.2008. Fuente: WINGCHING-JONES et al. (2009).

Tabla 1. Peso comparativo, área de apoyo y presión estática de animales en pastoreo, hombres y mujeres (GREENWOOD y McKENZIE, 2001). Adaptada de TABOADA (2007).

\begin{tabular}{lccc} 
& Masa & $\begin{array}{c}\text { Área total } \\
\text { superficial }\end{array}$ & $\begin{array}{c}\text { Presión } \\
\text { estática }\end{array}$ \\
\cline { 2 - 4 } & $\mathbf{K g}$ & $\mathbf{c m}^{2}$ & $\mathbf{k P a}$ \\
\hline Ovinos & $40-54$ & $55-84$ & $48-83$ \\
\hline Vacunos & $306-612$ & $264-460$ & $98-192$ \\
\hline Equinos & $400-700$ & 736 & $54-95$ \\
\hline Hombre & $61-75$ & $146-173$ & $41-45$ \\
\hline Mujer & $46-73$ & $45-79$ & $57-108$ \\
\hline
\end{tabular}

Debe considerarse que estas presiones aumentan durante el pastoreo, pues al caminar los animales sólo apoyan dos o tres de sus pezuñas. Por otra parte, la cantidad de tránsito dependerá mucho de la disponibilidad de forraje, y la distancia a los bebederos. Sin embargo, debe considerarse que un vacuno o un ovino pueden estar transitando hasta unas 12 - 13 horas por día.

Respuesta del suelo al tránsito animal: La respuesta del suelo al tránsito por animales depende del contenido hídrico que posee cuando es pastoreado. Esta influencia del pisoteo es principalmente de tipo físicomecánico, pues afecta el estado de compactación de los primeros centímetros del suelo. Cuando el suelo está más seco, el tránsito y pisoteo causan compactación del suelo, asociada con una pérdida de macroporosidad. La compactación deja escasa evidencia visual de su daño (Fig. 2). En cambio, el pastoreo en altas condiciones de humedad edáfica, da lugar a la ocurrencia de daño por "poaching". Este daño es causado por el flujo de suelo alrededor de la pezuña del animal, dejando una huella bien definida, o en condiciones de extrema humedad, un suelo totalmente amasado.
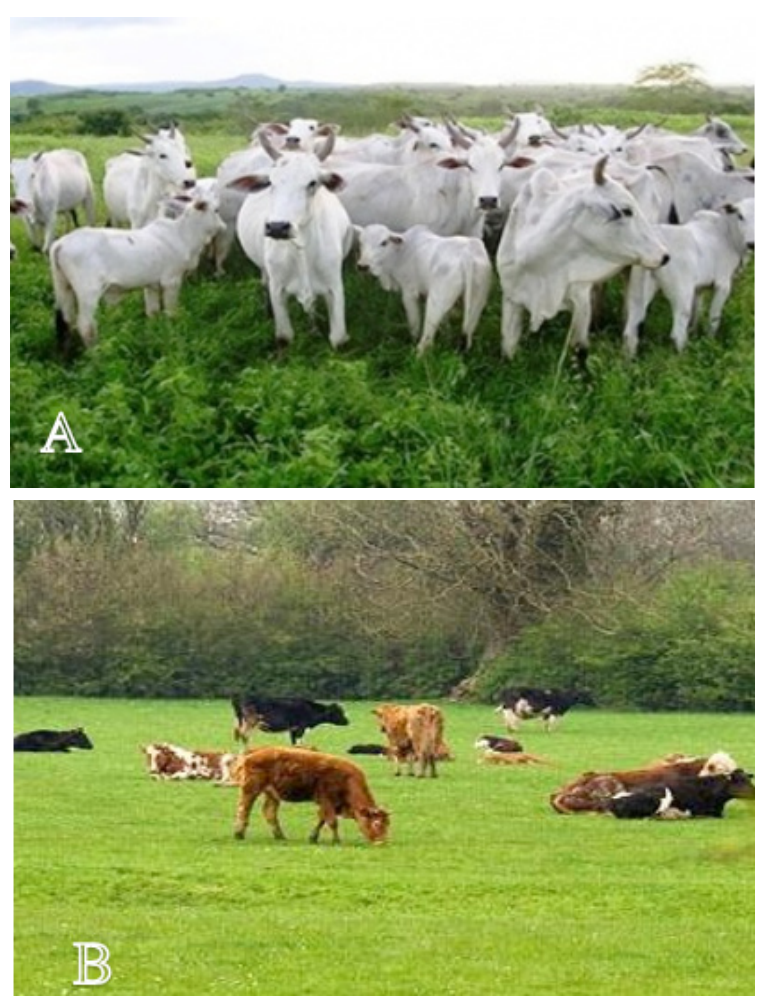

Figura 2. Tipos posibles de Ganadería (A. Intensiva, B. Extensiva.). Fuente: URL:http://www. contextoganadero.com/blog/ganaderia-intensivavs-ganaderia-extensiva; $B$.

Procesos de compactación: La Fig. 3 presenta, en forma idealizada, la secuencia de procesos que tienen lugar cuando un suelo es pastoreado con bajo o con alto contenido de humedad.

Cuando el suelo está próximo a la sequedad, su capacidad portante es máxima, y de este modo, la probabilidad de que sufra un daño estructural es mínima. En cambio, cuando el suelo está húmedo, su capacidad portante es menor, y se vuelve propenso a sufrir una 
Cuando un suelo es susceptible a sufrir daños estructurales?

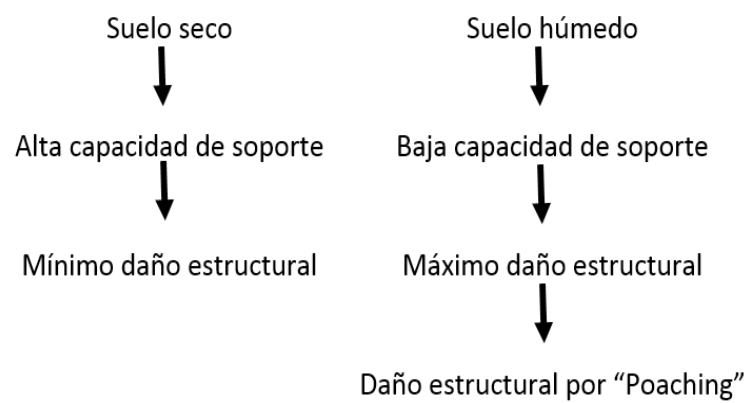

Figura 3. Esquema teórico que predice la influencia de la humedad edáfica sobre el desarrollo de daños estructurales por pisoteo. Fuente: TABOADA ( 2007).

mayor compactación superficial. Esto no es otra cosa que la deformación superficial del suelo superficial para soportar el peso del animal. Esta deformación se hace a expensas del espacio de macroporos.

Debido a ello, todo daño por compactación implica necesariamente la ocurrencia simultánea de:

- Aumentos de densidad aparente

- Descensos de macroporosidad

- Aumentos de resistencia superficial

- Descensos de infiltración y conductividad hidráulica saturada.

La acción mecánica de la pezuña animal causa la pulverización de los agregados del suelo, causando a menudo también pérdidas de estabilidad estructural. Estos daños suelen ser superficiales, tal como lo demuestra resultados de Taboada y Lavado (1988). Al comparar suelos no pastoreados con suelos pastoreados en forma continua, puede observarse que se producen aumentos significativos de densidad aparente y de resistencia a la penetración (Tabla 2).

Se observa que los aumentos de densidad aparente ocurrieron sólo en una fecha (noviembre), y que afectaron sólo los primeros ocho centímetros del suelo. Estos aumentos fueron acompañados por aumentos altamente significativos de resistencia superficial entre situaciones no pastoreadas y pastoreadas. Sin embargo, es fácil observar que la principal fuente de variación de la resistencia fue entre fechas, asociado con los cambios de humedad edáfica.

Estos cambios de densidad y resistencia estuvieron asociados con cambios en la macroporosidad (TABOADA y LAVADO, 1993). Precisamente, los poros del suelo responsables de la aireación y la entrada de agua al suelo.
Tabla 2. Densidad aparente y resistencia a la penetración de suelos no pastoreados y pastoreados, en diferentes fechas (TABOADA y LAVADO, 1988).

\begin{tabular}{|cccc|}
\multicolumn{1}{c}{} & \multicolumn{2}{c|}{ Densidad aparente (Mg/m3) } \\
\hline Fechas & Capa & No pastoreado & Pastoreado \\
\hline \multirow{3}{*}{ Nov } & $0-4 \mathrm{~cm}$ & $0.96^{*}$ & 1.00 \\
& $4-8 \mathrm{~cm}$ & $1.20^{*}$ & 1.29 \\
& $8-12 \mathrm{~cm}$ & 1.28 & 1.30 \\
\hline \multirow{4}{*}{ Junio } & $4-8 \mathrm{~cm}$ & 1.23 & 0.99 \\
& $8-12 \mathrm{~cm}$ & 1.29 & 1.24 \\
\hline
\end{tabular}

\begin{tabular}{crc} 
& \multicolumn{2}{c}{ Resistencia a la Penetración } \\
\cline { 2 - 3 } Sechas & No pastoreado Pastoreado \\
\cline { 2 - 3 } Nov & $450^{* *}$ & 940 \\
Dic & $470^{* *}$ & 690 \\
& $3710^{* *}$ & 6440
\end{tabular}

Remediación de los suelos degradados en su estructura

Mediante Herramientas Mecánicas: Para romper las capas compactadas en la temporada seca, cuando el suelo está muy duro, pueden ser necesarias herramientas robustas diferentes de aquellas que el agricultor normalmente usa para la labranza, tales como, la piocha, la piqueta, la azada de tres picos o una barreta larga.

Tradicionalmente, los métodos mecánicos son usados para romper las capas limitantes del crecimiento de las raíces, naturalmente densas o compactadas, con el fin de crear poros más grandes a través de los cuales puedan penetrar las raíces. Esto es hecho, generalmente, levantando ligeramente el implemento y rompiendo la capa densa o compactada.

Las capas poco profundas limitantes del crecimiento de las raíces, como los pisos de azada se encuentran típicamente de 5 a $8 \mathrm{~cm}$ de profundidad; el medio más fácil para romperlos es con subsoladores tirados por bueyes o arados de cincel montados sobre tractor (Fig. 4).

Las capas más profundas limitantes del crecimiento de las raíces, tales como los pisos de arado, se forman a partir del límite inferior de labranza del suelo lo que, por lo general, ocurre en los $20-25 \mathrm{~cm}$ de su perfil superior. 


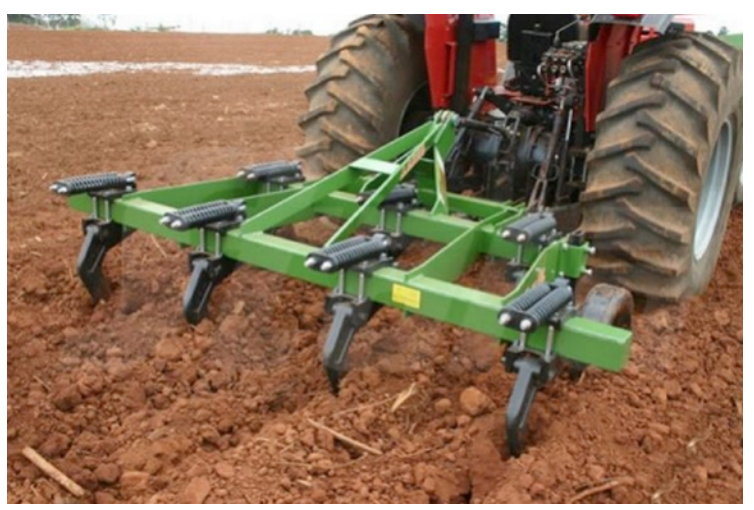

Figura 4. Subsolador montado en un tractor, usado para romper capas compactadas. Fuente: URL http:// www.viarural.cl/agricultura/maquinaria-agricola/ stara/asa-h-02.jpg.

Remediaciones por métodos biológicos: El método principal de restauración de la porosidad de las capas limitantes del crecimiento de las raíces es utilizar las raíces de la vegetación natural o de cultivos de cobertura plantados para actuar como subsoladores biológicos que penetren en los horizontes densos. La estabilidad de los canales creados por las raíces de las plantas será mayor que aquella de canales formados por métodos mecánicos, ya que la liberación de sustancias orgánicas de las raíces estabiliza las superficies internas de los canales. Una vez que las raíces se han muerto y contraído, estos poros serán lo suficientemente grandes y estables para permitir que penetren las raíces del cultivo siguiente.

La tierra puede ser dejada en barbecho por 2 - 3 años para su regeneración con arbustos naturales o vegetación forestal. Alternativamente, la siembra de especies seleccionadas efectivas en la regeneración de la estructura del suelo puede enriquecer el barbecho natural. Un cultivo de cobertura puede ser sembrado para que sirva como un barbecho sembrado. Las especies promisorias de cultivos de cobertura que han dado muestras de ser potenciales subsoladores biológicos son el pasto Bahía (Paspalum notatum), Festuca elatior, el pasto Guinea (Panicum maximum), la alfalfa (Medicago sativa), el gandul (Cajanus cajan) y el caupí (Vigna unguiculata).

El rábano forrajero (Raphanus sativus) y los arbustos fijadores de nitrógeno Tephrosia vogelii, Sesbania sesban y Gliricidia sepium han sido también identificados como potencialmente útiles. Algunas malezas con raíces principales fuertes, como Amaranthus spp., pueden tener potencial para actuar como subsoladores biológicos, como han observado los agricultores Mennonitas de Bolivia que encontraron rendimientos más altos en suelos compactados después de infestaciones fuertes de Amaranthus spp. (Fig. 5).

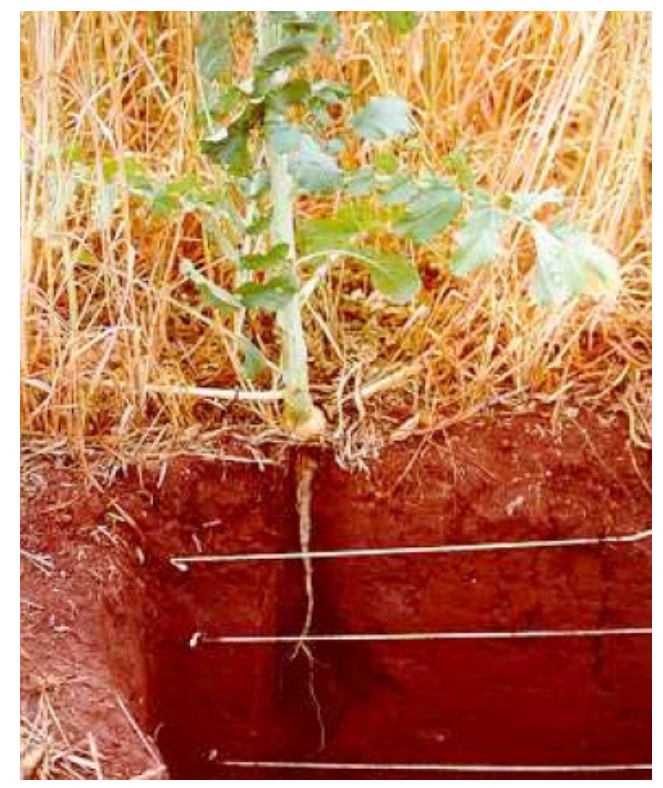

Figura 5. Ejemplo de una raíz muy visible de rábano penetrando en el suelo. El tamaño de la raíz principal indica lo beneficioso del rábano como «herramienta» biológica de descompactación. Cuando se practica la AC, esta raíz descompuesta dejará un bioporo en el suelo a través del cual el agua de lluvia podrá fácilmente infiltrarse. A. Calegari. Fuente: URL:http://www.fao.org/ag/ca/Training Materials/ CD27-Spanish/se/soil_assessment.pdf.

Los métodos biológicos son generalmente mucho más económicos de ejecutar y sus beneficios son de más larga duración que los métodos mecánicos. Una ventaja importante de los barbechos vegetativos es que mejoran sensiblemente la fertilidad física, química y biológica del suelo debido a la gran cantidad de materia orgánica producida y agregada al suelo. Los barbechos de árboles pueden ser beneficiosos suministrando leña, materiales de construcción y otros productos, siempre que la recolección de estos materiales no reduzca los efectos beneficiosos del barbecho sobre la fertilidad química del suelo

\section{Conclusiones}

En general, el pisoteo de los animales compacta el suelo en los primeros $15 \mathrm{~cm}$, ocasionando una severa disminución en el movimiento interno del agua y un aumento en la Densidad Aparente; esto trae como consecuencia una disminución en la porosidad y cambios desfavorables en la relación suelo-agua-aire que afecta el desarrollo de las raíces de las plantas y su productividad.

El pastoreo directo por ganado doméstico ejerce dos tipos de efectos sobre los suelos: a) la defoliación 
causada por la ingesta de forraje por los animales; y b) el pisoteo causado por el tránsito de ganado por los campos. Ambos efectos pueden generar importantes cambios en las propiedades físicas y químicas de los suelos, entre los que están una disminución apreciable en la productividad de los mismos.

Las soluciones a los problemas de compactación deben nacer de una evaluación de campo para conocer si hay o no capas compactadas, o adensadas y a qué profundidades éstas se encuentran.
Las remediaciones a los efectos de la degradación de los suelos por compactación se pueden realizar mediante la utilización de herramientas y procedimientos mecánicos, y también mediante métodos biológicos, pero en cualquiera de los casos, deberán sujetarse a la normatividad ambiental y con criterios de la agricultura sostenible, en el marco de la conservación y restauración de los recursos naturales. (FAO, Investment Centre Division).

\section{Referencias}

AMÉZQUITA, E. 1998. Hacia la sostenibilidad de los suelos de los Llanos Orientales. IX Congreso Colombiano de la Ciencia del Suelo, Paipa-Boyacá, Colombia, octubre 21- 24,1998.

AMÉZQUITA, C.E.; CHÁVEZ, O.L.F. 1999. La compactación del suelo y sus efectos en la productividad de los suelos. Congreso Costarricense de la ciencia del suelo, San José, Costa Rica, julio 1999.

DOUGLAS, M.G.; MUGHOGHO, S.K.; SAKA, A.R.; SHAXSON, T.F.; EVERS, G. 1999. Report on an investigation into the presence of a cultivation hoe pan under smallholder farming conditions in Malawi. Investment centre division FAO/World Bank cooperative programme. Washington, D.C. World Bank.

PINZÓN, A.; AMÉZQUITA, E. 1991. Compactación de suelos por pisoteo de animales en pastoreo en el piedemonte amazónico de Colombia. Revista Pasturas tropicales 13 (2):21-26.

TABOADA, M.A. 2007. Efectos del pisoteo y pastoreo animal sobre suelos en siembra directa. $4^{\circ}$ Simposio de Ganadería en Siembra Directa:1-13

WINGCHING-JONES, R.; CABALCETA-AGUILAR, G.; ALVARADO-HERNÁNDEZ, A. 2009. Impacto del pastoreo con ganado Holstein y Jersey sobre la densidad aparente de un andisol. Agronomía mesoamericana 20 (2):371-379.

SOANE, B.D. and C. y OUWERKERK. 1994. Soil compaction problems in world agriculture. P'gas. 1-21. En: Soane, B.D.; Ouwerkerk, C. Soil compaction in crop production. Países Bajos. Elsevier Science.

Conservación de los recursos naturales para una Agricultura sostenible. Disponible en: URL:http://www.fao.org/ag/ ca/Training Materials/CD27-Spanish/se/soil assessment.pdf. Consultado: 10-01-2015.

Ganadería intensiva vs. Ganadería extensiva. Disponible en: URL:http://www.contextoganadero.com/blog/ganaderiaintensiva-vs-ganaderia-extensiva. Consultado: 05-01-2015.

Ganadería extensiva para conservar el futuro Parque de Guadarrama. Disponible en: URL:http//www. diariodegastronomia.com/ganaderia-extensiva-para-conservar-el-futuro-parque-de-guadarrama/. Consultado: 0501-2015. 